\title{
Optical Design for Spectrograph with Coaxial Illumination
}

\author{
Jing-Heng Lan, Xian-Gyu Zeng, Ming-Hao Gao and Bai-Cheng Li* \\ University of Shanghai for Science and Technology, School of Optical-Electrical and Computer Engineering, \\ No. 580 Jungong Road, Shanghai, 200093, China
}

(Received February 9, 2019; revised version March 6, 2019; in final form March 8, 2019)

\begin{abstract}
As an important optical instrument, spectrograph is widely used to analyze material components and some characteristics through spectral analysis, such as chemical research, medial detection, food industry, geological detection and other fields. Different spectrographs shall be chosen in different application fields. In this study, a transmission spectrograph was designed to detect spectrum of light scattered on an object surface. Working distance of this spectrograph was $30 \mathrm{~mm}$ and a coaxial illumination system was used in this spectrograph. Such illumination scheme eliminates reflected light rays of the objective lens and specular reflection light rays on the testing object surface by using polarization beam splitter, which increases the accuracy of the instrument. A LED was applied as the lighting source, so the instrument is characterized by low power consumption, quick response, and long service life. The designed spectrograph is optimized generally by CODE V10.4 with appropriate initial parameters and aspherical lens. The detection range and spectral resolution of the spectrograph are $400-780 \mathrm{~nm}$ and $0.4 \mathrm{~nm}$, respectively.
\end{abstract}

DOI: 10.12693 /APhysPolA.136.182

PACS/topics: coaxial illumination, optical design, spectrograph design, spectral resolution, LED illumination

\section{Introduction}

Spectrograph is extensively used in various fields, such as chemical research [1-3], medical detection [4], food industry and geological prospecting [5]. The basic principle of spectrograph is to break up light into separate colours by using the dispersion element [6]. However, the requirements on parameters of the spectrograph are variable in different application fields. With the rapid development and expanding application range of scientific technology, the requirements on size, performance, and price of spectrograph are proposed.

Spectrograph can be generally divided into refractive type and reflective type according to the internal light path style. The reflective type is superior since it is free of chromatic aberration, and has smaller size than the refractive type. Therefore, most spectrographs on the market are reflective type. However, with the development of aspherical lens processing technology, shortages of reflective type spectrograph can be reduced and coaxial light illumination can be realized. More and more refractive spectrographs will be developed in future. In this study, a refractive spectrograph was designed to detect spectra of scattered light on object surface. The working distance of the designed spectrograph is $30 \mathrm{~mm}$. This spectrograph carries coaxial light illumination system and eliminates reflected light rays on the objective lens and specular light on the object by using a polarizing beam splitter. Moreover, LED as the illumination light source, causes the spectrograph to have characteristics such as low power consumption, quick response, and long service life. The overall optimization of the designed

\footnotetext{
*corresponding author; e-mail: shhf201382@163.com
}

spectrograph was realized by using CODE V10.4 [7] with appropriate initial parameters and aspherical lens. The detection range and spectral resolution of the spectrograph were $400-780 \mathrm{~nm}$ and $0.4 \mathrm{~nm}$, respectively. Finally, stray light was analyzed by Lighttools 8.4.

\section{System principles and initial structural calculation}

Design requirements of this study are shown in Table I. Due to the requirement of high spectral resolution, a grating must be used as the dispersion element. Due to the different bidirectional scattering distribution function (BSDF) of different objects, the use of coaxial illumination can make the sampling more consistent. In order to retain the uniformity of sampling, attentions need to be paid to the used mode of coaxial light source. Moreover, the entrance pupil of the imaging light path is in the same plane as the measured surface. To realize coaxial illumination, the imaging light path and illumination light path share one objective lens. Two light paths are coupled through the splitter. The designed light paths is shown in Fig. 1.

Design requirements

TABLE I

\begin{tabular}{l|c}
\hline \hline \multicolumn{1}{c|}{ Parameter } & Value \\
\hline spectral range & $400-780 \mathrm{~nm}$ \\
spectral resolution & $0.4 \mathrm{~nm}$ \\
working distance of lens & $40 \mathrm{~mm}$ \\
region of detection & $\emptyset 4 \mathrm{~mm}$ \\
illumination mode & coaxial illumination \\
power & $<1 \mathrm{~W}$ \\
size (maximum) & $100 \times 100 \times 16 \mathrm{~mm}$
\end{tabular}




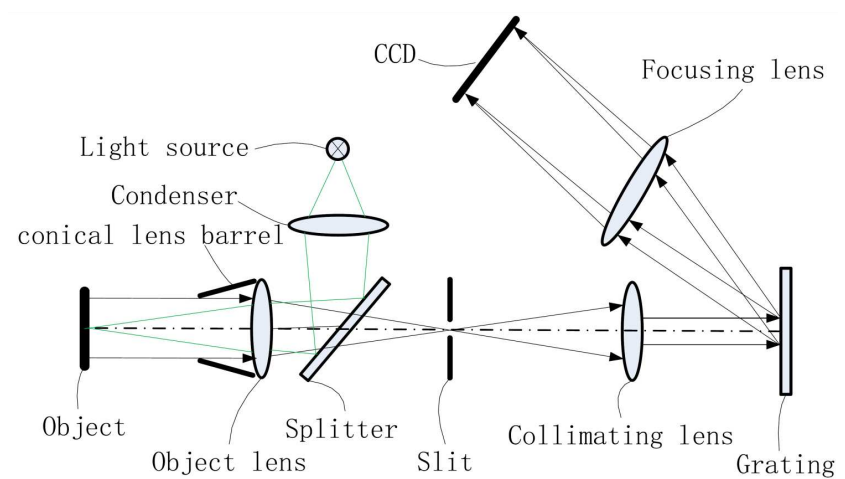

Fig. 1. Light paths.

The principle of illumination path is: the light emitted from the light source run through the condenser and then is split by the splitter. The reflected light split by the splitter is focused on the object surface through the object lens. The principle of imaging path is: light after diffuse reflection by the measured surface is converged on the slit through the object lens. Slit serves as the spatial filter removing reflected light from outside the detection area of the measured object. The collimating lens collimate light through the slit and make it incident onto the grating vertically. A reflecting plane grating is used. For the same order of diffraction, different wavelengths of light are reflected at different angles. Focusing lens focus the light of diffraction order $m=1$ (or $m=-1$ ) onto CCD.

There is a design requirement of $30 \mathrm{~mm}$ working distance of spectrograph systems. In order to reduce stray light of the environment, a conical lens barrel should be added to the front of the objective lens. The length of the barrel was designed to be $10 \mathrm{~mm}$, the entrance pupil distance was designed as $40 \mathrm{~mm}$, and the entrance pupil diameter was selected as $4 \mathrm{~mm}$. To reduce stray light formed by reflected light by the lens surface, the object lens needs to be designed as achromatic doublet. According to experiences, the achromatic doublet can achieve good image quality in a small field of view with $f / 5.6$. Therefore, the focal length of object lens was determined preliminarily as $f_{O}^{\prime}=4 \times 5.6=22.4 \mathrm{~mm}$.

According to the design requirements, resolution of the spectrograph has to reach $0.4 \mathrm{~nm}$. According to the chromatic resolution of the grating

$$
\Delta \lambda=\frac{\lambda}{m N D_{G}},
$$

where $\Delta \lambda$ is the minimum wavelength difference for grating to be distinguished, $m$ is the diffraction order, $N$ is the number of grating lines, and $D_{G}$ is the width of grating. The common blazed grating with 1000 lines $/ \mathrm{mm}$ was applied. Then, $N=1000, m=1, \Delta \lambda=0.4$, and $\lambda=780$ were put into Eq. (1), to obtain the minimum value of $D_{G}$, which was $1.95 \mathrm{~mm}$. To realize a better effect, the beam diameter incident onto the grating was $D=2.5 \mathrm{~mm}$. The relative aperture of collimat- ing lens should be the same as that of object lens. The focal length was $f_{C}^{\prime}=2.5 \times 5.6=14 \mathrm{~mm}$. Diameter of the collimating lens $\left(D_{C}\right)$ should not be lower than the beam diameter incident onto the grating $(D)$. For the convenience of processing and assembly, it was set as $D_{C}=6 \mathrm{~mm}$ in the present study.

Light propagates through the collimating lens and enter onto the grating plane perpendicularly. Therefore, the diffraction angle of different wavelengths can be gained according to the grating equation

$$
d \sin \theta=m \lambda m=0,1,2 \ldots,
$$

where $d$ is the grating constant, $\theta$ is the diffraction angle, $m$ is the diffraction order, and $\lambda$ is the wavelength. In this design, $d=1 / N=1000 \mathrm{~nm}, m=1, \lambda_{0}=400 \mathrm{~nm}$, $\lambda_{1}=780 \mathrm{~nm}$. These parameters can be brought into Eq. (2) to calculate the half-field angle of the spectral diffraction: $\omega=\frac{\theta_{1}-\theta_{0}}{2}=13.84^{\circ}$.

It reaches a resolution of $0.4 \mathrm{~nm}$ in the wavelength range of 400-780 $\mathrm{nm}$. Therefore, $(780-400) / 0.4=950$ pixels of CCD are needed, at least. In this design, the specification of $1024 \times 1$ pixels was used as the linear array CCD. Each pixel size was $14 \mu \mathrm{m} \times 14 \mu \mathrm{m}$ and the length of light-sensitive surface was $14.336 \mathrm{~mm}$. Therefore, we can determine that the focal length of focusing lens as $f_{F}^{\prime}=\frac{L}{2 \tan \omega}=\frac{14.336}{2 \times \tan 13.84^{\circ}} \approx 29.1 \mathrm{~mm}$.

For the distance between collimating lens and grating $\left(L_{C G}\right)$, it can be determined as $L_{C G}>\frac{D+D_{C}}{2 \tan \theta_{0}}=$ $\frac{2.5+6}{2 \tan \theta_{0}}=9.17 \mathrm{~mm}$ from the angle of the diffraction order $m=1$ (or -1) in the $400 \mathrm{~nm}$ wavelength, beam diameter on the grating $(D)$, and diameter of collimating lens $\left(D_{C}\right)$. With consideration to the installation space of focusing lens and space of other structural components, we can determine $L_{C G}$ as $20 \mathrm{~mm}$. Figure 2 is a schematic diagram of calculating $L_{C G}$.

Based on preliminary calculation of the system in this section, the general initial model of the designed spectrograph was established. The detailed design of the lens is introduced in the following section.

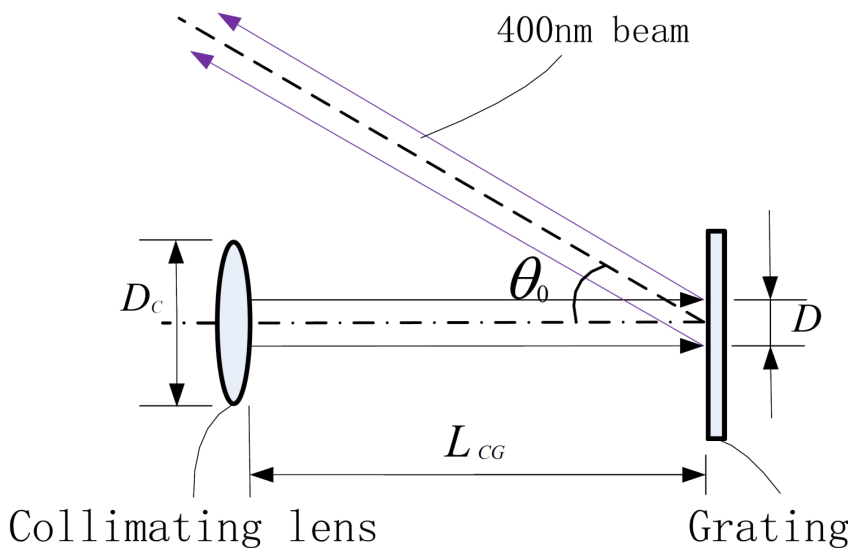

Fig. 2. Schematic diagram of calculating $L_{C G}$. 


\section{Lens design}

\subsection{Design of the imaging lens}

Splitter has to be considered before designing the object lens. Splitter is the equivalent to parallel plate in the imaging path and it would not produce any curvature of field under any conditions. However, splitter will produce some aberration since it is not in the parallel path. In this design, the field of view of the imaging system is relatively small, resulting in the small astigmatism, distortion, and chromatic difference of magnification. There are large spherical aberration and axial chromatic aberration in the large relative aperture optical system. Therefore, aberration of splitter, especially chromatic aberration, shall be considered during the process of designing. These aberrations of splitter are proportional to its thickness and increases with the increase of refraction index. In order to obtain enough mechanical strength, thickness of the splitter was set as $0.16 \mathrm{~mm}$ and the splitter was made of N-BK7.

The initial structure of objective lenses was calculated by the PW method. (A method of acquiring an initial structure of an optical system by "aberration characteristic parameters".) Next, the thickness of lens was selected according to processing experiment in CODE V10.4. A splitter was added and the focal length was set to $22.09 \mathrm{~mm}$ by automatic optimization.

For illumination splitting, the splitter was put at an inclined angle of $45^{\circ}$. Therefore, the splitter produced offaxis aberration. To compensate this off-axis aberration, eccentricity setting in the collimating lens was needed. The collimating lens was designed in the same way as object lens. The collimating lens was a doublet lens with a focal length of $14 \mathrm{~mm}$.

The focusing lens plays a crucial role of improving the resolution of spectrograph. Therefore, aspherical focusing lens was used to correct aberrations except curvature of field and chromatic aberration. The rotationally symmetric surface with a sag is the most common aspheric surface, which is defined as:

$$
z=\frac{c r^{2}}{1+\sqrt{1-(1+k) c^{2} r^{2}}}+\sum_{i=1}^{n} a_{i} r^{2 i}
$$

where $z$ is the sag of the surface parallel to the z-axis, $c$ is the base curvature at the vertex, $k$ is a conic constant, $r$ expresses the radial coordinate measured perpendicularly from the optical axis, and $a_{i} r^{2} i$ denote the higher-order aspheric terms. Aspherical lenses are easily machined by using single-point diamond lathes.

Light paths of the imaging system are shown in Fig. 3. The object lens and collimating lens form a Keplerian telescope, with the middle image at the slit. This Keplerian telescope is composed of two positive components spaced so that the second focal point of the first component coincides with the first focal point of the second. This component is independently aberration corrected and can be modularly assembled in the future. The eccentricity of focusing lens is relatively small and rotary symmetric lens can be made easily which decreases the difficulty of processing effectively. The system performance can be analyzed from the disc of confusion at the image surface.

It can be seen from Fig. 4 that sizes of spots in different wavelengths are lower than $0.01 \mathrm{~mm}$, which are smaller than the pixel size of CCD. Therefore, the design of this imaging system can reach the resolution of $0.4 \mathrm{~nm}$. Moreover, the resolution can be further increased by using CCD with smaller pixel size.

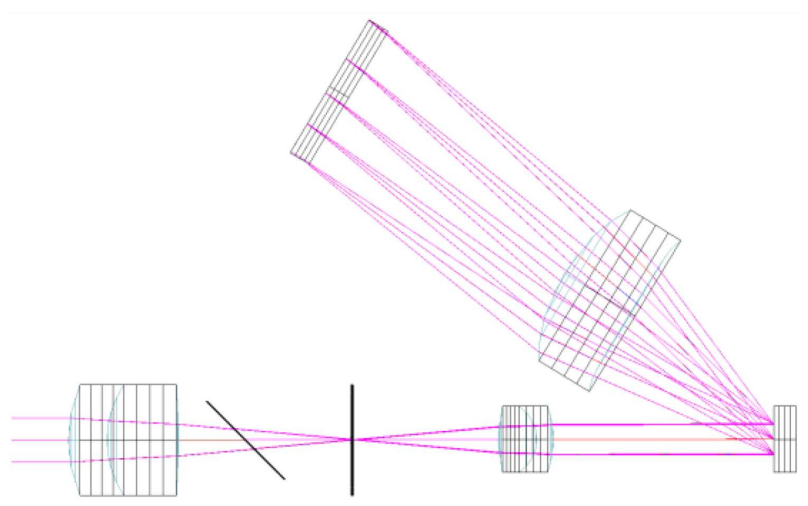

Fig. 3. Light paths of the imaging system.

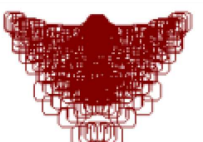

WL: 780nm

size : $0.00849 \mathrm{~mm}$

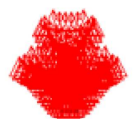

WL: 700nm

size : $0.00587 \mathrm{~mm}$

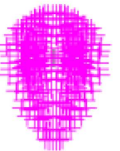

WL: $600 \mathrm{~nm}$

size : $0.00783 \mathrm{~mm}$

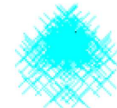

WL: 500nm

size : $0.00396 \mathrm{~mm}$

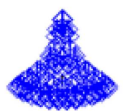

WL: 400nm

size : $0.00626 \mathrm{~mm}$

\section{$0.05 \mathrm{~mm}$}

Fig. 4. Shape and size of spot. 


\subsection{Design of illuminating lens}

Before designing illumination lens, the type of light source has to be determined. LED has characteristics of quicker response, longer service life, and smaller volume than other electric light sources. However, halogen lamp, gas discharge lamp, and laser, as light sources, were used instead of LED as the spectral width of LED with wide spectrum is not gentle and extensive like halogen lamp and gas discharge lamp in the past. Usually the spectral energy of the LED is extremely uneven. However with the study of sunlike source some LED of full visible spectrum has been developed. In this design, LED light source in spectrograph is applied with STW9C2PB$\mathrm{S}(\mathrm{CW})$ of Sunlike series of SEOUL SEMICONDUCTOR because the spectral curve of $\mathrm{CW}(\mathrm{CW} / \mathrm{NW} / \mathrm{WW}$ represents 3 types of LEDs: cool white, neutral white, warm white) is more gradual than the other. Its power and the size of light-emitting area are $0.2 \mathrm{~W}$ and $3.0 \times 3.0 \mathrm{~mm}$, respectively. The spectral curve is shown in Fig. 5 .

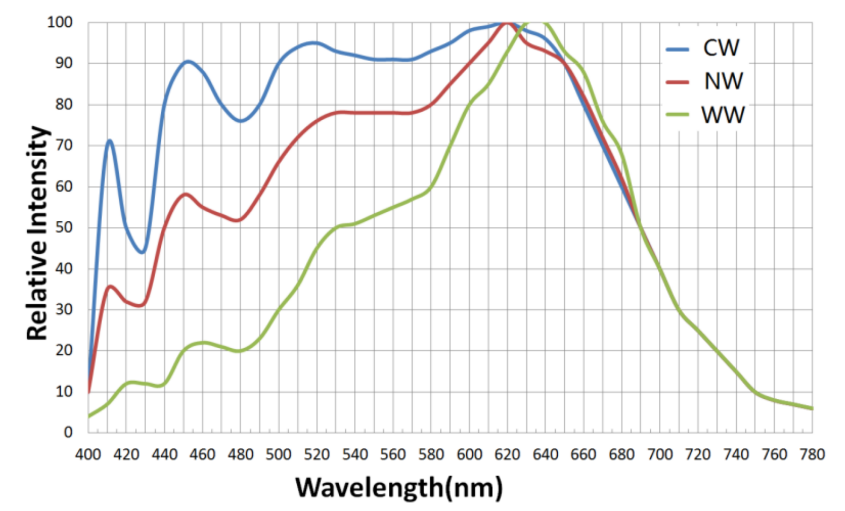

Fig. 5. Spectral curve of LED.

It can be seen from Fig. 5 that the spectral curve of CW is relatively gentle in the range of $410-680 \mathrm{~nm}$, and the fluctuation in the range of $400-780 \mathrm{~nm}$ is also acceptable.

The illumination system used the Kohler illumination [8] which image the light source onto pupil entrance of the imaging system, thus enabling gain of high illumination uniformity. Therefore, it is necessary to design an illumination system to conjugation of luminous surface of the light source and surface of the object. To avoid influences of aberration of the illumination system on failure of the spectrograph system, the size of lighting spot has to be larger than the entrance pupil diameter of the imaging system. In this case, the illuminated spot diameter was $3.5-4.0 \mathrm{~mm}$. Size of the luminous surface of the chosen LED was close to $3.0 \times 3.0 \mathrm{~mm}$. LED can be treated as a Lambertian source. With comprehensive considerations to influencing factors of aberration and stray light, the magnification power of the illumination system was 0.5 .

Illumination light paths are shown in Fig. 6, which are in inverse design. The object surface is the "object" in the optical system, while the luminous surface of LED

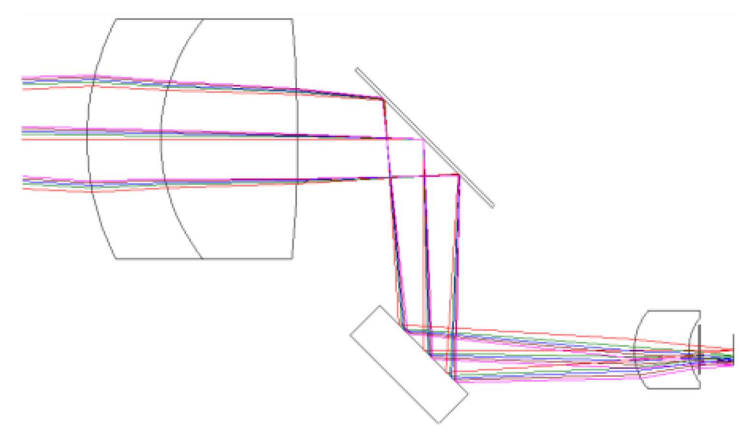

Fig. 6. Illumination light paths.

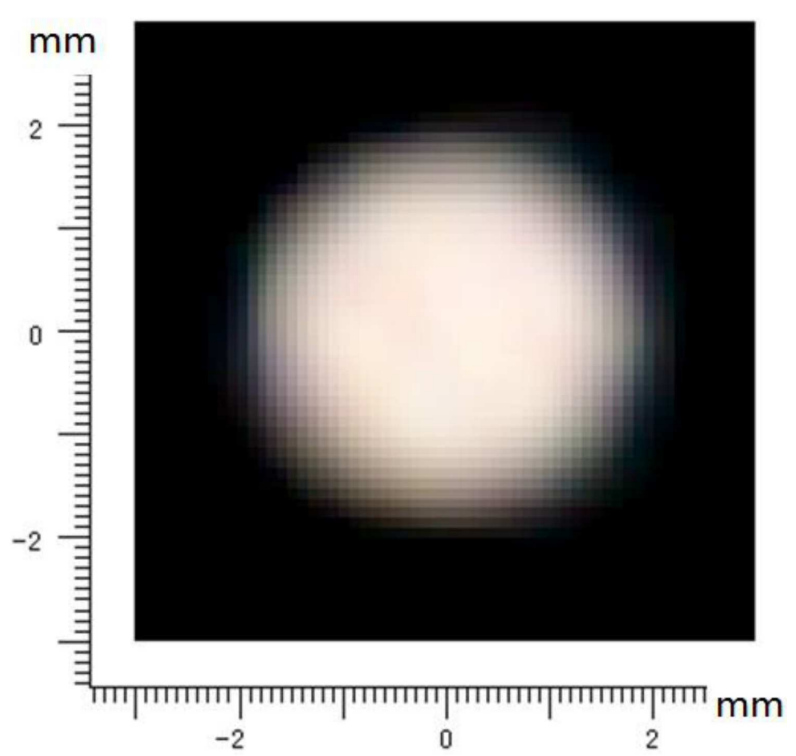

Fig. 7. Effect of the illumination system.

is the "image". Finally, field stop is added before shining LED to restrict the imaging size. The final effect of illumination could be simulated by Lighttools8.4 (Fig. 7).

It can be seen from Fig. 7 that uniform illumination can be realized in the region of $\emptyset 3.5 \mathrm{~mm}$, indicating that the illumination system meets the design expectation.

\section{Stray light analysis and simulation}

In this design, the stray light source of the spectrograph is mainly composed of three parts: (1) reflection by optical surface of object lens, (2) reflected light rays of large aperture on the object surface, (3) light on the grating 0 -th order and minus 1-st order and other high orders. For the elimination of stray lights, reflectivity of structural components is decreased. In addition, it optimizes in the following ways.

Since the reflectivity of the measured object is relatively low, attention has to be paid to the reflected light of object lens. Except for the AR film coating, the internal straight light is decreased by using polarizing beam splitting. The principle is shown in Fig. 8. 


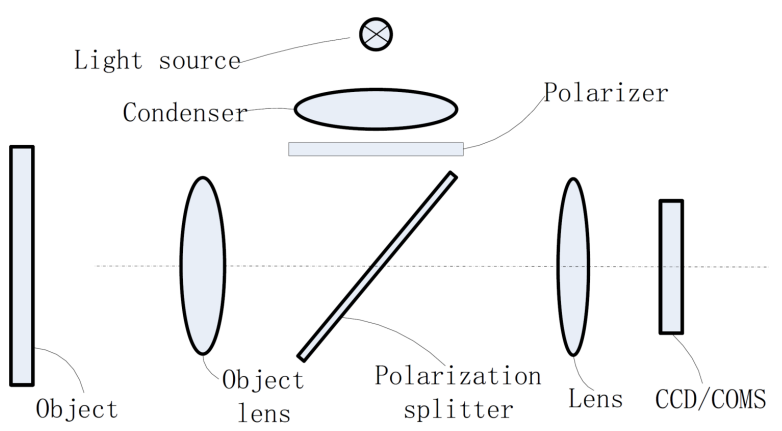

Fig. 8. Principle of stray light elimination of polarizing beam splitting.

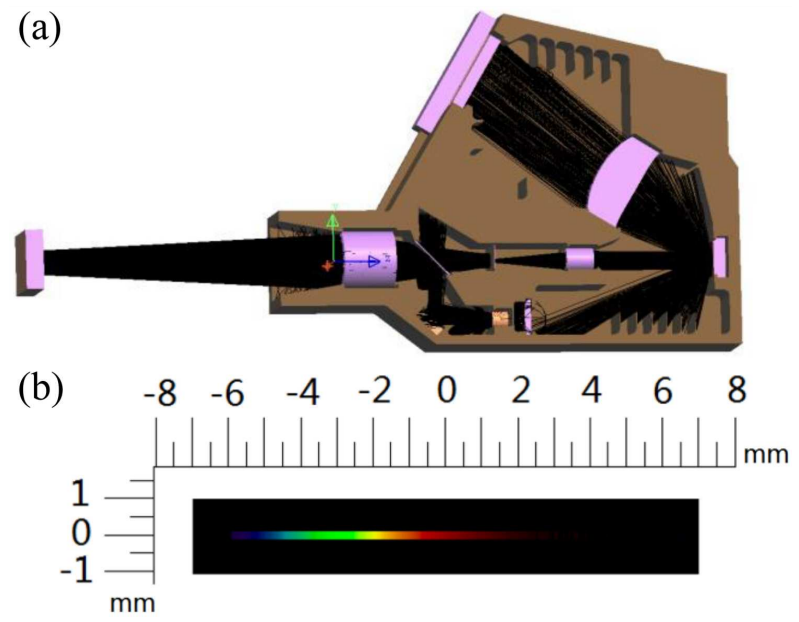

Fig. 9. Lighttools model: (a) model graph, (b) CCD simulation.

Light from the light source was collected by the condenser. It then entered onto the polarizer, forming S polarizing light. The polarization splitter transmits $\mathrm{P}$ light and reflects $\mathrm{S}$ light. Light reflected by the object may be polarizing light which is mainly composed of $\mathrm{S}$ light. Most of the $\mathrm{S}$ light is reflected by polarization splitter. Therefore, it fails to arrive at CCD, decreasing internal stray light in the system significantly.

Light reflected from the large aperture on the object surface can be eliminated significantly by controlling the size of slit accurately and by adding the field stop close to collimating lens.
For lights reflected from 0-order grating, on the one hand, most reflected light can be transferred to other orders by designing the grating blaze angle. On the other hand, 0-order reflected orders would return basically along the original light path, thus decreasing influences of grating 0-order reflected light rays.

For light rays from high-order diffraction grating, stray light rays can be decreased using multiple reflections by changing the angle of incidence of the light onto the structural components.

The Lighttools model of the spectrograph and true color simulation image of CCD are shown in Fig. 9a and $b$, respectively.

\section{Conclusions}

A small spectrograph with coaxial illumination system is designed based on the plane diffraction grating in this study. LED is used as the light source. It can reach $0.4 \mathrm{~nm}$ of spectral resolution in the range of 400-780 $\mathrm{nm}$. By using the polarizing extinction method and opticalmechanical structure design, stray light is analyzed and eliminated, which increases accuracy of the spectrograph. The final size of spectrograph is $78 \times 56 \times 13 \mathrm{~mm}$, realizing the goal of small size. The idea and approach of this design provide references for further increase of spectral resolution and mini-size design.

\section{References}

[1] Y.V. Knyazev, A.V. Lukoyanov, Y.I. Kuzmin, A.G. Kuchin, M. Vasundhara, Opt. Spectrosc. 124, 784 (2018).

[2] N.L. Thomas, S.A. Johnson, C.G. Stevens, Proc. SPIE 2266, (1994).

[3] D. Khmelevskaya, D.P. Shcherbinin, E.A. Konshina, M.M. Abboud, A. Dubavik, I. A. Gladskikh, Opt. Spectrosc. 125, 731 (2018).

[4] T. Vo-Dinh, Eng. Med. Biol. Mag. IEEE 23, 40 (2004).

[5] H. Weihong, D.W. Sun, J. Blasco, Postharvest Biol. Technol. 125, 168 (2017).

[6] G. Lemaitre, Astron. Astrophys. 103, L14 (1981).

[7] D. Hasenauer, Synopsys White Paper: Choosing the Right Optical Design Software, 2018.

[8] D.S. Goodman, A.E. Rosenbluth, Optical/Laser Microlithography Proc. SPIE 922, 108 (1988). 